\title{
T Cell Targeted Therapies in Lupus: Do They Make Sense?
}

\author{
Aikaterini Thanou, $M D^{1, *}$ \\ Joan Merrill, $M D^{2}$
}

\author{
Address \\ ${ }^{*}, 1$ Arthritis and Clinical Immunology Research Program, Oklahoma Medical \\ Research Foundation, 825 NE 13th Street, MS 22, Oklahoma City, OK 73104, USA \\ Email: aikaterini-thanou@omrf.org \\ ${ }^{2}$ Clinical Pharmacology Research Program, Oklahoma Medical Research \\ Foundation, 825 NE 13th Street, MS 22, Oklahoma City, OK 73104, USA
}

Published online: 4 January 2015

(C) Springer International Publishing AG 2015

\section{This article is part of the Topical Collection on Lupus}

Keywords Systemic lupus erythematosus - T cell • Th17 • Tf • Costimulation • CD40L • CTLA4 - ICOS • mTOR • IL6 • IFNY $\cdot \mathrm{N}$-acetylcysteine $\cdot$ Laquinimod $\cdot$ Paquinimod $\cdot$ Forigerimod

\section{Opinion statement}

Although systemic lupus erythematosus (SLE) is well-characterized as a B cell-driven, autoantibody mediated disease, recent studies focusing on innate immunity and on the interacting roles of other cell types, including T cells, have illuminated a wider scope of pathophysiology. The development of effective targeted therapies for SLE has been limited by the complex nature of these multiple interacting immune pathways. T cells in SLE display an activated phenotype with increased responses by effector subsets coupled to deficient regulatory responses. This singular SLE phenotype has been associated with specific intracellular signaling changes such as activation of the NFAT pathway and altered transcription of characteristic immune mediating proteins, including those related to IL6, IL23, and IL17 pathways. Some promising treatments developed to specifically regulate these pathways are currently in preclinical or early clinical development and a few have moved on to later phase trials. We propose that a specific currently identifiable subset of patients with SLE (those with altered TH17/Treg responses and/or elevated IL6 and/or BLyS signals) are likely the best candidates for treatment with appropriately targeted T cell modulating agents. Although this remains to be proven, our opinion is that advances in SLE treatment will only occur when there is a better understanding of pre-treatment biology both to optimize patient selection and to provide markers for improving dosing strategies. It may be that strategic combinations of targeted agents tailored to individual patterns of immune activation will provide the best approach to treatment in the future. 


\section{Introduction}

Systemic lupus erythematosus (SLE) is a systemic autoimmune disease characterized by a deregulated immune response to self-antigens with widespread production of autoantibodies and tissue injury [1]. T cells play a complex role in SLE. Although they engage B cells to promote B cell maturation and classic autoantibody production, these are actually co-stimulatory interactions, leading to alterations in the phenotypes of both $\mathrm{B}$ and $\mathrm{T}$ cells, which contributes to immune activation through multiple effector mechanisms and decreased regulatory activity. T cells in patients with SLE display a hyperactive phenotype with a net alteration in transcription of multiple immune-regulating proteins. These immune pathways represent promising targets for $\mathrm{T}$ cell directed therapies in SLE, as has been demonstrated in vitro and in animal models of the disease. This review will summarize some key pathophysiologic abnormalities in SLE T cells with a focus on their relatedness to investigational treatments targeting $\mathrm{T}$ cells that are undergoing clinical trials.

\section{T cell subsets in SLE}

T cells are categorized by a range of effector and regulatory phenotypes based on the expression of transcription factors and cytokines [2]. Alterations in frequencies and functions of $\mathrm{T}$ cell subpopulations, with increased effector and decreased regulatory responses, are well characterized in SLE [3, 4] In addition to the classical Th1 and Th2 lineages, attention has been drawn to increased signals from Th17 cells, both in animal models and humans with SLE [5].

Serum levels of IL17 and the prevalence of Th17 cells are increased in patients with SLE and have been correlated to disease activity in some studies [6]. Double negative (CD4-CD8-) T cells are also expanded in the peripheral blood of patients with SLE and represent, along with Th17 cells, a major source of IL17 [7]. Overproduction of IL17 is implicated in lupus pathology by promoting recruitment and activation of other inflammatory cells that cause tissue injury [5]. Renal infiltration of IL17 producing cells has been demonstrated in patients with lupus nephritis and in lupus prone mice [7]. IL17 can also act in synergy with BLyS in stimulation of B cell survival, differentiation, and autoantibody production, ultimately leading to amplification of the autoimmune response $[8,9 \bullet]$ Effector CD4+ T cells localized in B cell follicles (follicular helper T cells) are important for B cell survival, via signaling through CD40L, PD1, and IL21. These cells promote germinal center formation, somatic hypermutation of B cells, and plasma cell differentiation [10]. Studies in animal models provide evidence of the role of follicular helper T cells (Tfh) in SLE pathogenesis, where abrogation of their development or function was found to be therapeutically beneficial [10].

In addition to upregulated effector $\mathrm{T}$ cell responses, SLE patients have reduced numbers and impaired function of CD4+ regulatory T cells (Treg) [11]. An imbalance between Th17 cells and Treg, with higher ratio of Th17 to Treg cells compared to healthy controls has also been reported in SLE [12•]. IL6, a pleiotropic cytokine secreted by $\mathrm{T}$ cells, monocytes, endothelial cells, and fibroblasts, has been implicated in this imbalance, by means of promoting Th17 differentiation and suppressing FoXP3 transcription, thus eventually leading to reduction in Tregs [13•]. 
Thus, agents that could enhance the numbers and function of Tregs without causing widespread immune activation could, at least in theory, be therapeutically beneficial, but are not yet tested in SLE. In the interim, therapeutic inhibition of classic cytokines promoting T-cell functions, such IL-6, IL-21, and IFN- $\gamma$ has been explored in SLE, with promising early phase studies [14, 15] Agents targeting other T cell-related cytokines, such as IL-17 and IL-12/IL23 , have been studied in malignancies, psoriasis, inflammatory bowel disease, and/or rheumatoid arthritis, but are not in the development for SLE at this time. In a small open-label study of tocilizumab, a humanized monoclonal antibody to the IL6 receptor administered to 16 patients with mild to moderately active SLE, disease activity significantly improved after 12 weeks, with reduction in inflammatory markers and anti-double-stranded DNA antibody levels [16]. Improvements however occurred to the expense of dose-related neutropenia and a high rate of infections, likely due to global IL6 blockade by inhibition of both soluble and membrane-bound receptors [13•]. Several other IL6 targeting therapies are in development, including antibodies to IL6 and a soluble gp130IgFc fusion protein that exclusively targets the IL6/sIL6-R signaling pathway [13•]. Sirukumab (CNTO 136) a fully human monoclonal antibody to IL6 successfully completed a phase I safety and pharmacokinetics study in systemic lupus [17] and is currently on a phase II RCT in lupus nephritis [NCT01273389]. Another fully human anti-IL6 antibody, PF-04236921, has completed a phase II trial in patients with systemic lupus [NCT01405196], the results of which are likely to become available in the near future. A randomized controlled blinded dose-escalation study of NNC0114-0006, a monoclonal antibody to IL21, is underway in SLE [NCT01689025].

\section{Surface and intracellular signaling in SLE T cells}

Following engagement of the T cell receptor (TCR), T cells from patients with SLE display rapid and enhanced calcium influx and phosphorylation of a wide range of cytoplasmic proteins [18]. These alterations have been linked to TCR rewiring, with replacement of $\mathrm{CD} 3 \zeta$ by FcR $\gamma$ and subsequent recruitment of Syk instead of the canonical ZAP-70 kinase [15]. Syk, an ubiquitously expressed kinase of the Src family, has a central regulatory role in SLE, affecting protein and gene expression of a number of key pathogenic molecules, including IL21 and CD44 [19]. CD44 is involved in cell adhesion and migration and is increased SLE T cells, likely facilitating $\mathrm{T}$ cell migration to inflamed tissues [19]. Another mechanism that may contribute to enhanced TCR-mediated signaling in SLE is the clustering of lipid rafts, which are areas of the T cell membrane where signaling molecules tend to accumulate [15].

Intracellular transmission of signals initiated on the T cell surface culminates in transcription factor activation and modification of gene expression. The robust calcium influx in SLE T cells following activation leads to increased calcineurin and NFAT activation and enhanced transcription of several early response genes [20]. CAMK4 is activated by the increased calcium response and in turn activates the transcription factor CREM and the mammalian target of rapamycin (mTOR), a pivotal serine/threonine-protein kinase of the outer mitochondrial membrane $[15,21]$. CREM inhibits transcription of IL2, which is important for Treg activation, whereas mTOR controls $\mathrm{T}$ cell activation by 
multiple metabolic pathways (including $\mathrm{Ca}^{+2}$ movement and maintenance of the mitochondrial transmembrane potential) and enhances Th17 differentiation [22]. Another transcription factor, CREB, acts opposite to CREM when phosphorylated, increasing IL2 transcription [23]. Phosphorylated CREB is inactivated by PP2A, a phosphatase that is also regulated by CAMK4 and has increased activity in SLE [23]. These interacting pathways have been reviewed in detail elsewhere [23] and provide a particularly promising avenue for T cell directed therapies, the development of which is expected to expand.

Identification of key signaling molecules in SLE T cells, like Syk, CAMK4, and ROCK, resulted in successful proof-of-principle studies of their inhibitors in murine lupus, with potential to prove useful in humans with SLE. Preclinical studies of agents targeting these molecules have been reviewed elsewhere [15], and here, we will only focus agents targeting the calcineurin/NF-AT signaling that are currently in clinical trials. The calcineurin inhibitor cyclosporine A was as effective as cyclophosphamide in a trial of sequential induction and maintenance therapy in patients with proliferative lupus nephritis [24]. Tacrolimus, a macrolide antibiotic that also inhibits calcineurin, was found to be more effective and safer than intravenous cyclophosphamide in induction treatment for Asians with proliferative or membranous lupus nephritis [25], [26]. Longterm tacrolimus treatment was also well tolerated and effective, especially in regards to reducing proteinuria, in a series of 29 Asian patients with lupus nephritis, but larger prospective studies including other racial groups are warranted [27]. A topical formulation of the calcineurin inhibitor pimecrolimus was comparable to betamethasone $0.1 \%$ cream in a double-blind randomized controlled trial of facial discoid lupus [28]. Systemic administration of cyclosporine and calcineurin inhibitors can be limited by side effects, most notably nephrotoxicity and induction of diabetes. Dipyridamole, an inhibitor of platelet aggregation used for stroke prevention with an excellent safety profile, was found to selectively inhibit calcineurin signaling. Dipyridamole decreased CD40L expression, IFN $\gamma$, IL17, and IL6 production and immunoglobulin secretion in SLE T cells in vitro and in a murine model alleviated lupus nephritis while preventing the appearance of skin ulcers [29].

\section{T cell costimulation in SLE}

Activated T cells in patients with SLE abundantly express CD154, the ligand for CD40 on antigen presenting cells (also known as CD40 ligand or CD40L). Evidence from animal models and humans supports an essential role of the CD40-CD40L interactions in generation of pathogenic autoantibodies and tissue injury in SLE [30]. Activation of the CD40 pathway leads to upregulation of costimulatory and adhesion molecules, permitting production of proinflammatory cytokines (such as IL1, TNF $\alpha$, and IL6), antibody class switching, and B cell affinity maturation [31]. Soluble CD40L is increased in patients with SLE, and has been found to track with disease severity or activity in different studies $[32,33]$

Despite promising preclinical data, a phase II double-blind randomized controlled trial of IDEC-131, a humanized monoclonal antibody to CD40L administered in 85 patients with SLE did not show significant benefits in disease activity [34]. Multiple factors might have confounded these results, 
including the observed high placebo responses, the heterogeneous distribution of disease activity at study entry, the use of variable doses of the study medication and the relatively short duration of treatment [34]. A subsequent openlabel study of another monoclonal antibody to CD40L, BG9588 in 28 patients with proliferative lupus nephritis failed to provide further insights into therapeutic efficacy, and was stopped prematurely due to thromboembolic events [35]. This could be due to the fact that platelets of patients with SLE strongly express CD40L on their surface and can be cross-linked via binding of the antiCD40L Fc to platelet Fc $\gamma$ RII $\alpha$ receptor, potentially leading to platelet aggregation and thromboembolism [36]. Anti-CD40L constructs that do not trigger Fc $\gamma$ RII $\alpha$ signaling, such as single IgG molecules and domain antibodies, could bypass platelet activation-related toxicities while maintaining their biological efficacy [31]. CDP7657, a monovalent PEGylated Fab portion of an anti-CD40L antibody is currently on a phase I study in SLE [NCT01093911].

Variations in T cell expression or function of CTLA4, an endogenous inhibitor of the CD28/B7 costimulatory pathway, have also been implicated in SLE pathogenesis [37]. Although CTLA4 expression is increased in effector CD4+ T cells of patients with SLE, CTLA4 is excluded from membrane microdomains and unable to inhibit $\mathrm{T}$ cell proliferation or regulate intracellular pathways [38]. Nonetheless, binding of soluble CTLA4 to B7 can inhibit activating signals between B7 and CD28, a rationale for the development of abatacept (CTLA4Ig) as a circulating inhibitor of B7 interactions. Although abatacept showed promising results in preclinical studies in SLE and in patients with rheumatoid arthritis, two randomized controlled trials in patients with SLE with [39] and without [40] renal manifestations did not meet their primary or secondary end points. In post hoc analysis of the nonrenal trial, a treatment effect of abatacept in flare prevention was suggested for the subgroup of patients able to taper their background steroids to low levels in the last 3 months of the study [40]. Another post hoc analysis suggested differences between abatacept and placebo for flares defined by physicians (clinically significant disease) or severe flares (as measured by a BILAG A score), and these differences appeared to be more pronounced among patients with polyarthritis at study entry. Similarly, an exploratory analysis of the dataset for the abatacept nephritis study was performed applying response criteria utilized in other lupus nephritis trials, and this analysis suggested potential efficacy of the medication for this indication [41]. However, an additional trial (The ACCESS study) was recently reported. Abatacept added to low dose intravenous cyclophosphamide did not improve complete renal response rates at 6 months compared to cyclophosphamide alone [42]. This combination treatment has been supported by data in murine lupus nephritis [43] and is also employed in the clinical trial of RG2077, a CTLA4-IgG4Fc fusion protein, that is underway in lupus nephritis [NCT00094380].

Another costimulatory interaction between ICOS on activated T cells and NK cells, with its ligand (ICOS ligand) is critical for a variety of T cell responses. ICOS ligand (also known as B7-related protein-1 or B7RP1) is constitutively expressed on B cells and monocytes, and promotes the development of Th17 and Tfh cells [10]. Overexpression of ICOS has been demonstrated in T cells of patients with SLE, enhancing their proliferation and ability to produce IFN $\gamma$, whereas antibodies to B7RP1 attenuated autoimmunity in murine lupus [44]. A humanized monoclonal antibody to B7RP1 (AMG557) has completed a phase I randomized placebo controlled trial in SLE [NCT00774943]. 


\section{Oxidative stress in SLE T cells}

T cells in patients with SLE exhibit mitochondrial hyperpolarization, increased production of reactive oxygen species and diminished antioxidant defenses upon exposure to nitric oxide, that have been attributed to profound depletion of glutathione (GSH) and loss of endogenous antioxidant enzyme activities [45•]. The resulting increase in reactive oxygen intermediates predisposes cells to necrosis and is associated with disease activity and target organ damage in SLE [45•]. Reactive oxygen species are essential modulators of various signal transduction pathways, including production of regulatory microRNA, control of mRNA translation and protein folding, as well as the degradation and recycling of proteins and organelles via autophagy. Such a pivotal signal transductor activated by oxidative stress is the mTOR kinase of the outer mitochondrial membrane, a key regulator of $\mathrm{T}$ cell proliferation and differentiation [22]. The activity of mTOR is increased in lupus $\mathrm{T}$ cells and has been implicated in the pathogenic lupus $\mathrm{T}$ cell cytokine overexpression and Treg contraction $[21,46]$. Furthermore, decreased DNA methylation and altered histone acetylation induced by oxidative stress in SLE T cells can contribute to SLE pathogenesis through effects on transcription patterns and ultimately $\mathrm{T}$ cell signaling and lineage development [47]. In accordance with these diverse functions, oxidative stress mediates T-cell dysfunction in SLE at multiple levels and could be an important focus of targeted therapies [45•].

Rapamycin (sirolimus), a macrolide inhibitor of the mTOR kinase, was shown to alleviate some features of SLE refractory to other immune suppressants in a small open label study of nine patients [48]. Rapamycin prolonged survival, normalized proteinuria, and reduced anti-dsDNA titers in lupus-prone mice, while ameliorating histological lesions and Akt/mTOR glomerular expression [49]. Mechanistic studies have uncovered multiple distinct mechanisms of action of rapamycin in SLE that are mediated by inhibition of mTORC1, including the suppression of IL17 expression by CD4+T cells and the expansion of Tregs [46]. N-acetylcysteine (NAC), a precursor of glutathione (GSH) and a stimulator of its de novo synthesis, can effectively raise GSH levels in lymphocytes, thus regulating the mitochondrial transmembrane potential and the activity of mTOR. In a double blind randomized placebo-controlled pilot study in 36 patients with SLE, NAC safely improved disease activity and fatigue within 3 months, while profoundly blocking mTOR and expanding regulatory T cells [50]. NAC appears to be a safe, inexpensive, mechanismdriven approach that warrants further investigation in larger studies.

\section{Other T cell modulatory therapies}

Laquinimod, an orally administered small molecule of the quinoline-3carboxamide family that was originally developed for the treatment of multiple sclerosis, was found to be equivalent to mycophenolate mofetil in preventing and suppressing murine lupus nephritis [51]. Laquinimod benefits survival, proteinuria, and glomerulonephritis in mice by effects on both myeloid and lymphoid immune cells [51]. In a randomized controlled trial in patients with lupus nephritis [NCT01085097], laquinimod combined with standard of care 
was well tolerated and demonstrated an additive (although not statistically significant) benefit in renal function over 24 weeks compared to standard of care [52]. Results of a phase IIa trial of laquinimod in lupus arthritis [NCT01085084] are anticipated. Another quinoline-3-carboxamide derivative, paquinimod (ABR-215757), was well tolerated in a phase Ib dose-ranging RCT in 20 patients with SLE and low disease activity [53]. In a small open-label study of paquinimod added on standard therapy in 13 patients with mildly active SLE and mucocutaneous and/or musculoskeletal involvement, improvement in arthritis, oral ulcers, and/or alopecia was seen over 12 weeks [54].

Forigerimod [Lupuzor (IPP- 201101)] is a 21-amino-acid fragment of U1 snRNP 70 kDa phosphorylated at Ser140, which is thought to inhibit autophagy and produce altered ligation of the $\mathrm{T}$ cell receptor, modulating the autoreactive T cell phenotype $[55,56]$. In a randomized, controlled trial of 149 South American patients with SLE active on standard of care treatment, forigerimod was well tolerated and more effective than placebo [57]. The response rate using the SLE responder index (SRI) was $53.1 \%$ in forigerimodtreated patients at 12 weeks versus $36.2 \%$ in the placebo arm $(p=0.048)$. In a sub-analysis restricted to patients with more active disease at entry (defined by SLEDAI score $\geq 6$ ), the response rate using SRI increased to $61.9 \%$ in the treatment versus $38.6 \%$ in the placebo group $(p=0.016)$ [57]. The drug is currently in phase III development. Of note, a formulation of the same peptide in trehalose instead of mannitol excipient did not meet its end points in a trial of similar design, reaching only a $34 \%$ SRI response rate at 24 weeks, compared with $40 \%$ in the placebo arm [58]. This discrepancy has been attributed to enhancement of autophagy by trehalose, counteracting the immunomodulatory properties of forigerimod [59]; however, this remains to be proven.

\section{Effects of therapies targeting other immune pathways on T cell activation in SLE}

Intertwining signals between $\mathrm{T}$ cells and other pathways of immune activation that are central in SLE pathogenesis, e.g., BlyS and Type I IFN pathways have been recently described. For example, type I IFN activity has been associated with Th17 responses and IL-17 production in SLE, e.g., by inducing production of cytokines such as IL- 6 that promote Th17 cell differentiation [9•]. IFN $\alpha$ stimulates Th1 responses, prevents apoptosis of cytotoxic T cells and suppress Tregs, thereby augmenting T cell effector functions. Therapies targeting the IFN $\alpha$ production and functions are expected to indirectly influence $\mathrm{T}$ cell responses and have been a significant focus of clinical research in SLE, with several potential treatments completing phase II clinical trials [60].

Induction of Th1 responses and IFN $\gamma$ production that occur in a subset of lupus patients may culminate in a more chronic, later stage organ inflammation, and fibrosing complications [61]. IFN $\gamma$, a pleotropic type II IFN that activates macrophages, NK cells, and cytotoxic T cells and can modulate the Th and Treg cellular functions, has been implicated in lupus pathogenesis in animal models and humans. Expression of IFN $\gamma$ inducible genes was increased in monocytes from patients with SLE [61]. AMG 811, a fully human monoclonal antibody to IFN $\gamma$ has evolved to a phase Ib, randomized, dose-escalation 


\section{Conclusion}

study in SLE patients with or without glomerulonephritis [NCT00818948]. A phase I study in patients with discoid lupus is also ongoing [NCT01164917].

BLyS, a ligand of the tumor necrosis family predominantly produced by myeloid cells, is a B cell targeted cytokine considered crucial for autoreactive B cell survival in SLE. Some data suggests that BLyS receptors are also expressed on $\mathrm{T}$ cells, suggesting that BLyS may also serve as a T cell costimulator [62]. In murine collagen-induced arthritis, BlyS supports Th17 cell proliferation, in turn amplifying IL-17 levels and, in full circle, its effects on B cells [63]. Several therapies targeting membrane and soluble BLyS its receptors are in advanced phases of development in SLE, and these are reviewed elsewhere [62].

The multifactorial pathophysiology of complex diseases such as SLE has posed a formidable barrier to treatment development. Elucidation of patterns in this immune disorder could help to improve the selection and dosing of targeted treatments. T cells in SLE display a characteristic phenotype with increased activation of effector cells and decreased activation of regulatory cells. Treatments that are currently in development have the potential to address this imbalance. Further study of a newly characterized subset of SLE patients who share disordered Th17/Treg responses and activation of the IL6 and BLyS pathways might help to determine the optimal use of these new agents.

\section{Compliance with Ethics Guidelines}

\section{Conflict of Interest}

Aikaterini Thanou declares that she has no conflict of interest.

Joan Merrill has received consulting fees from Biogen Idec, Bristol-Meyers Squibb and AMG. She has also received grant funding to institution from Pfizer, Bristol-Meyers Squibb, Exagen and Glaxo-Smith Kline. She has received honoraria from Glaxo-Smith Kline, Research Pharmaceutical Services, UCB, Amgen, Pfizer, and Eli Lilly. She has been a consultant for Genetech/Roche, EMD Serono, Neovacs, Cephalon, Medimmune, Takeda, Macrogenics, Kirin, Questcor, Argos, Abbott, Ono, Astellas, Baxter, RPS, Parexel, Eisai, Incyte, and Novo Nordisk.

\section{Human and Animal Rights and Informed Consent}

This review article does not contain any previously unpublished studies with human or animal subjects performed by any of the authors.

\section{References and Recommended Reading}

Papers of particular interest, published recently, have been highlighted as:

- Of importance

1. Liu Z, Davidson A. Taming lupus-a new understanding 2. of pathogenesis is leading to clinical advances. Nat Med. 2012;18(6):871-82.

Palmer MT, Weaver CT. Autoimmunity: increasing suspects in the CD4+ T cell lineup. Nat Immunol. 2010;11(1):36-40. 
3. Lit LC, Wong CK, Li EK, Tam LS, Lam CW, Lo YM. Elevated gene expression of Th1/Th2 associated transcription factors is correlated with disease activity in patients with systemic lupus erythematosus. J Rheumatol. 2007;34(1):89-96.

4. Dolff S, Bijl M, Huitema MG, Limburg PC, Kallenberg CG, Abdulahad WH. Disturbed Th1, Th2, Th17 and T (reg) balance in patients with systemic lupus erythematosus. Clin Immunol. 2011;141(2):197-204.

5. Shin MS, Lee N, Kang I. Effector T-cell subsets in systemic lupus erythematosus: update focusing on Th17 cells. Curr Opin Rheumatol. 2011;23(5):444-8.

6. Shah K, Lee WW, Lee SH, Kim SH, Kang SW, Craft J, et al. Dysregulated balance of Th17 and Th1 cells in systemic lupus erythematosus. Arthritis Res Ther. 2010;12(2):R53.

7. Crispin JC, Oukka M, Bayliss G, Cohen RA, Van Beek CA, Stillman IE, et al. Expanded double negative T cells in patients with systemic lupus erythematosus produce IL-17 and infiltrate the kidneys. J Immunol. 2008; 181(12):8761-6.

8. Doreau A, Belot A, Bastid J, Riche B, Trescol-Biemont $\mathrm{MC}$, Ranchin B, et al. Interleukin 17 acts in synergy with B cell-activating factor to influence B cell biology and the pathophysiology of systemic lupus erythematosus. Nat Immunol. 2009;10(7):778-85.

9. $\quad$ Ambrosi A, Espinosa A, Wahren-Herlenius M. IL-17: a new actor in IFN-driven systemic autoimmune diseases. Eur J Immunol. 2012;42(9):2274-84.

This paper reviews the role of Il17 in autoimmunity and the interacting roles of IL17 and type I IFN in sustaining and amplifying autoimmune responses.

10. Craft JE. Follicular helper T cells in immunity and systemic autoimmunity. Nat Rev Rheumatol. 2012;8(6):337-47.

11. Bonelli M. Quantitative and qualitative deficiencies of regulatory $\mathrm{T}$ cells in patients with systemic lupus erythematosus (SLE). Int Immunol. 2008;20:861-8.

12.• Alunno A, Bartoloni E, Bistoni O, Nocentini G, Ronchetti S, Caterbi S, et al. Balance between regulatory $\mathrm{T}$ and Th17 cells in systemic lupus erythematosus: the old and the new. Clin Dev Immunol.

2012;2012:823085.

Concise review of current knowledge on the interplay of Treg and Th17 cells in SLE.

13. Jones SA, Scheller J, Rose-John S. Therapeutic strategies for the clinical blockade of IL-6/gp130 signaling. J Clin Invest. 2011;121(9):3375-83.

This is a comprehensive review of IL6 mediated signaling and how it can be modulated therapeutically.

14. Ronnblom L, Elkon KB. Cytokines as therapeutic targets in SLE. Nat Rev Rheumatol. 2010;6(6):339-47.

15. Crispin JC, Kyttaris VC, Terhorst C, Tsokos GC. T cells as therapeutic targets in SLE. Nat Rev Rheumatol. 2010;6(6):317-25.

16. Illei GG, Shirota Y, Yarboro CH, Daruwalla J, Tackey E, Takada K, et al. Tocilizumab in systemic lupus erythematosus: data on safety, preliminary efficacy, and impact on circulating plasma cells from an open-label phase I dosage-escalation study. Arthritis Rheum. 2010;62(2):542-52.

17. Szepietowski JC, Nilganuwong S, Wozniacka A, Kuhn A, Nyberg F, van Vollenhoven RF, et al. Phase I, randomized, double-blind, placebo-controlled, multiple intravenous, dose-ascending study of sirukumab in cutaneous or systemic lupus erythematosus. Arthritis Rheum. 2013;65(10):2661-71.

18. Moulton VR, Tsokos GC. Abnormalities of T cell signaling in systemic lupus erythematosus. Arthritis Res Ther. 2011;13(2):207.

19. Grammatikos AP, Ghosh D, Devlin A, Kyttaris VC, Tsokos GC. Spleen tyrosine kinase (Syk) regulates systemic lupus erythematosus (SLE) T cell signaling. PLoS One. 2013;8(8):e74550.

20. Kyttaris VC, Wang Y, Juang YT, Weinstein A, Tsokos GC. Increased levels of NF-ATc2 differentially regulate CD154 and IL-2 genes in T cells from patients with systemic lupus erythematosus. J Immunol. 2007;178(3):1960-6.

21. Koga T. Hedrich CM. Mizui M. Yoshida N. Otomo K. Lieberman LA. et al. CaMK4-dependent activation of AKT/mTOR and CREM-alpha underlies autoimmunity-associated Th17 imbalance. J Clin Invest. 2014

22. Powell JD, Delgoffe GM. The mammalian target of rapamycin: linking $\mathrm{T}$ cell differentiation, function, and metabolism. Immunity. 2010;33(3):301-11.

23. Apostolidis SA, Lieberman LA, Kis-Toth K, Crispin JC, Tsokos GC. The dysregulation of cytokine networks in systemic lupus erythematosus. J Interferon Cytokine Res. 2011;31(10):769-79.

24. Zavada J, Pesickova S, Rysava R, Olejarova M, Horak P, Hrncir Z, et al. Cyclosporine A or intravenous cyclophosphamide for lupus nephritis: the Cyclofa-Lune study. Lupus. 2010;19(11):1281-9.

25. Deng J, Huo D, Wu Q, Yang Z, Liao Y. A meta-analysis of randomized controlled trials comparing tacrolimus with intravenous cyclophosphamide in the induction treatment for lupus nephritis. Tohoku J Exp Med. 2012;227(4):281-8.

26. Yang M, Li M, He W, Wang B, Gu Y. Calcineurin inhibitors may be a reasonable alternative to cyclophosphamide in the induction treatment of active lupus nephritis: a systematic review and meta-analysis. Exp Ther Med. 2014;7(6):1663-70.

27. Yap DY. Ma MK. Mok MM. Kwan LP. Chan GC. Chan TM. Long-term data on tacrolimus treatment in lupus nephritis. Rheumatol (Oxford). 2014

28. Barikbin B, Givrad S, Yousefi M, Eskandari F. Pimecrolimus $1 \%$ cream versus betamethasone 17 valerate $0.1 \%$ cream in the treatment of facial discoid lupus erythematosus: a double-blind, randomized pilot study. Clin Exp Dermatol. 2009;34(7):776-80.

29. Kyttaris VC, Zhang Z, Kampagianni O, Tsokos GC. Calcium signaling in systemic lupus erythematosus $\mathrm{T}$ cells: a treatment target. Arthritis Rheum. 2011;63(7):2058-66. 
30. Sidiropoulos PI, Boumpas DT. Lessons learned from anti-CD40L treatment in systemic lupus erythematosus patients. Lupus. 2004;13(5):391-7.

31. Xie JH, Yamniuk AP, Borowski V, Kuhn R, Susulic V, Rex-Rabe S, et al. Engineering of a novel anti-CD40L domain antibody for treatment of autoimmune diseases. J Immunol. 2014;192(9):4083-92.

32. Kato K, Santana-Sahagun E, Rassenti LZ, Weisman MH, Tamura N, Kobayashi S, et al. The soluble CD40 ligand sCD154 in systemic lupus erythematosus. J Clin Invest. 1999;104(7):947-55.

33. Goules A, Tzioufas AG, Manousakis MN, Kirou KA, Crow MK, Routsias JG. Elevated levels of soluble CD40 ligand (sCD40L) in serum of patients with systemic autoimmune diseases. J Autoimmun. 2006;26(3):165-71.

34. Kalunian KC, Davis Jr JC, Merrill JT, Totoritis MC, Wofsy D. Treatment of systemic lupus erythematosus by inhibition of T cell costimulation with anti-CD154: a randomized, double-blind, placebo-controlled trial. Arthritis Rheum. 2002;46(12):3251-8.

35. Boumpas DT, Furie R, Manzi S, Illei GG, Wallace DJ, Balow JE, et al. A short course of BG9588 (anti-CD40 ligand antibody) improves serologic activity and decreases hematuria in patients with proliferative lupus glomerulonephritis. Arthritis Rheum. 2003;48(3):719-27.

36. Robles-Carrillo L, Meyer T, Hatfield M, Desai H, Davila $\mathrm{M}$, Langer F, et al. Anti-CD40L immune complexes potently activate platelets in vitro and cause thrombosis in FCGR2A transgenic mice. J Immunol.

2010;185(3):1577-83.

37. Romo-Tena J, Gomez-Martin D, Alcocer-Varela J. CTLA-4 and autoimmunity: new insights into the dual regulator of tolerance. Autoimmun Rev.

2013;12(12):1171-6.

38. Jury EC, Flores-Borja F, Kalsi HS, Lazarus M, Isenberg DA, Mauri C, et al. Abnormal CTLA-4 function in T cells from patients with systemic lupus erythematosus. Eur J Immunol. 2010;40(2):569-78.

39. Furie R, Nicholls K, Cheng TT, Houssiau FA, BurgosVargas R, Chen SL. Efficacy and safety of abatacept over 12 months in patients with lupus nephritis: results from a multicenter, randomized, double-blind, placebo-controlled phase II/III study. Arthritis Rheum. 2011;63(10 Suppl):S962-3.

40. Merrill JT, Burgos-Vargas R, Westhovens R, Chalmers A, D'Cruz D, Wallace DJ, et al. The efficacy and safety of abatacept in patients with non-life-threatening manifestations of systemic lupus erythematosus: results of a twelve-month, multicenter, exploratory, phase IIb, randomized, double-blind, placebo-controlled trial. Arthritis Rheum. 2010;62(10):3077-87.

41. Wofsy D, Hillson JL, Diamond B. Abatacept for lupus nephritis: alternative definitions of complete response support conflicting conclusions. Arthritis Rheum. 2012;64(11):3660-5.

42. Rovin BH. Investigators TAT. Treatment of lupus nephritis with abatacept plus low-dose pulse cyclophosphamide: the results of the ACCESS trial. Am Soc Nephrol 2013; Suppl (24):1B

43. Cunnane G, Chan OT, Cassafer G, Brindis S, Kaufman E, Yen TS, et al. Prevention of renal damage in murine lupus nephritis by CTLA-4Ig and cyclophosphamide. Arthritis Rheum. 2004;50(5):1539-48.

44. Hu YL, Metz DP, Chung J, Siu G, Zhang M. B7RP-1 blockade ameliorates autoimmunity through regulation of follicular helper T cells. J Immunol. 2009;182(3):1421-8.

45. Perl A. Oxidative stress in the pathology and treatment of systemic lupus erythematosus. Nat Rev Rheumatol. 2013;9(11):674-86.

This is a comprehensive review of the pathogenesis of oxidative stress and its relevance to SLE.

46. Kato H, Perl A. Mechanistic target of rapamycin complex 1 expands Th17 and IL-4+ CD4-CD8- doublenegative T cells and contracts regulatory $\mathrm{T}$ cells in systemic lupus erythematosus. J Immunol. 2014;192(9):4134-44.

47. Patel DR, Richardson BC. Epigenetic mechanisms in lupus. Curr Opin Rheumatol. 2010;22(5):478-82.

48. Fernandez D, Bonilla E, Mirza N, Niland B, Perl A. Rapamycin reduces disease activity and normalizes $\mathrm{T}$ cell activation-induced calcium fluxing in patients with systemic lupus erythematosus. Arthritis Rheum. 2006;54(9):2983-8.

49. Stylianou K, Petrakis I, Mavroeidi V, Stratakis S, Vardaki E, Perakis K, et al. The PI3K/Akt/mTOR pathway is activated in murine lupus nephritis and downregulated by rapamycin. Nephrol Dial Transplant. 2011;26(2):498-508.

50. Lai ZW, Hanczko R, Bonilla E, Caza TN, Clair B, Bartos A, et al. N-acetylcysteine reduces disease activity by blocking mammalian target of rapamycin in T cells from systemic lupus erythematosus patients: a randomized, double-blind, placebo-controlled trial. Arthritis Rheum. 2012;64(9):2937-46.

51. Lourenco EV, Wong M, Hahn BH, Palma-Diaz MF, Skaggs BJ. Laquinimod delays and suppresses nephritis in lupus-prone mice and affects both myeloid and lymphoid immune cells. Arthritis Rheumatol. 2014;66(3):674-85.

52. Jayne D, Appel G, Chan TM, Barkay H, Weiss R, Wofsy D. A randomized controlled study of laquinimod in active lupus nephritis patients in combination with standard of care. Ann Rheum Dis. 2013;72 Suppl 3:164.

53. Bengtsson AA, Sturfelt G, Lood C, Ronnblom L, van Vollenhoven RF, Axelsson B, et al. Pharmacokinetics, tolerability, and preliminary efficacy of paquinimod (ABR-215757), a new quinoline-3-carboxamide derivative: studies in lupus-prone mice and a multicenter, randomized, double-blind, placebo-controlled, repeatdose, dose-ranging study in patients with systemic lupus erythematosus. Arthritis Rheum. 2012;64(5):1579-88.

54. Bengtsson A, Sturfelt G, Rönnblom L, Gunnarsson I, Svenungsson E, Jacobsen S, et al. An exploratory study 
to evaluate changes in disease activity and biomakers during treatment with ABR-215757 in patients with mild active systemic lupus erythematosus (SLE). Ann Rheum Dis. 2011;70 Suppl 3:316.

55. Schall N, Page N, Macri C, Chaloin O, Briand JP, Muller S. Peptide-based approaches to treat lupus and other autoimmune diseases. J Autoimmun. 2012;39(3):143-53.

56. Monneaux F, Hoebeke J, Sordet C, Nonn C, Briand JP, Maillere B, et al. Selective modulation of CD4+ T cells from lupus patients by a promiscuous, protective peptide analog. J Immunol. 2005;175(9):5839-47.

57. Zimmer R, Scherbarth HR, Rillo OL, Gomez-Reino JJ, Muller S. Lupuzor/P140 peptide in patients with systemic lupus erythematosus: a randomised, doubleblind, placebo-controlled phase IIb clinical trial. Ann Rheum Dis. 2013;72(11):1830-5.

58. Zimmer R, Wallace DJ, Muller S. Randomized, doubleblind, placebo-controlled studies of P140 peptide in mannitol (lupuzor) and trehalose (forigerimod) in patients with SLE. Arthritis Rheum. 2012;64 Suppl 10:S1110.

59. Muller S. Wallace D. The importance of implementing proper selection of excipients in lupus clinical trials. Lupus. 2014

60. Kirou KA, Gkrouzman E. Anti-interferon alpha treatment in SLE. Clin Immunol. 2013;148(3):303-12.

61. Karonitsch T, Feierl E, Steiner CW, Dalwigk K, Korb A, Binder $\mathrm{N}$, et al. Activation of the interferon-gamma signaling pathway in systemic lupus erythematosus peripheral blood mononuclear cells. Arthritis Rheum. 2009;60(5):1463-71.

62. Liu Z, Davidson A. BAFF and selection of autoreactive B cells. Trends Immunol. 2011;32(8):388-94.

63. Lai Kwan Lam Q, King Hung Ko O, Zheng BJ, Lu L. Local BAFF gene silencing suppresses Th17-cell generation and ameliorates autoimmune arthritis. Proc Natl Acad Sci U S A. 2008;105(39):14993-8. 